\title{
Los criterios de Rosemont en ultrasonografía endoscópica: ¿son predictores de insuficiencia pancreática exocrina?
}

\author{
Marianela Arramón, ${ }^{1}$ Agustina Redondo, ${ }^{1}$ Pablo Cura, ${ }^{1}$ Fernando Baldoni, ${ }^{1}$ Ana Brizio, ${ }^{2}$ \\ Cecilia Loudet, ${ }^{3}$ Francisco Tufare ${ }^{1}$ \\ ${ }^{1}$ Servicio de Gastroenterología, HIGA "San Martín de La Plata". \\ 2 Servicio de Laboratorio, HIGA "San Martín de La Plata". \\ ${ }^{3}$ Servicio Docencia e Investigación, HIGA "San Martín de La Plata". \\ La Plata, provincia de Buenos Aires, Argentina.
}

Acta Gastroenterol Latinoam 2021;51(2):189-195

Recibido: 19/06/2020 / Aceptado: 26/05/2021 / Publicado online: 21/06/2021 / https://doi.org/10.52787/qwpo5827

\section{Resumen}

La ultrasonografía endoscópica es el método de elección para la detección de cambios morfológicos relacionados a pancreatitis crónica. Existen, en la bibliografía, criterios que evalúan por ultrasonografía endoscópica cambios morfológicos sugestivos de pancreatitis crónica. Los más utilizados son los criterios de Rosemont. Actualmente, los métodos diagnósticos para insuficiencia pancreática exocrina de mayor eficacia son la cuantificación de grasa (en desuso) y el test de aire espirado que utiliza triglicéridos marcados con C13 (TG-C13), no disponible en nuestro pais; pero la probabilidad del diagnóstico de insuficiencia pancreática puede ser estimada sobre la base de sintomas, parámetros de malnutrición, elastasa en materia fecal y signos de pancreatitis crónica en imágenes. El

Correspondencia: Marianela Arramón Avenida $60 n^{\circ}$ 512, dpto. 11 B, La Plata, provincia de Buenos Aires, Argentina / Tel.: 2284218772

Correo electrónico: marianelaarramon@gmail.com objetivo del presente trabajo fue determinar la correlación, probabilidad y severidad de insuficiencia pancreática exocrina, de acuerdo con los criterios de Rosemont, en pacientes con diagnóstico de pancreatitis crónica. Se realizó un estudio retrospectivo y descriptivo. Se recolectaron datos de pacientes entre abril de 2017 y abril de 2018 en relación con las características morfológicas pancreáticas, de acuerdo con los criterios de Rosemont, y se relacionaron con el diagnóstico de insuficiencia pancreática exocrina de acuerdo con el nivel de elastasa en materia fecal. Se incluyeron veintiun pacientes con pancreatitis crónica. La presencia de focos hiperecogénicos $>$ de $2 \mathrm{~mm}$ con sombra acústica posterior se asoció a la presencia de insuficiencia pancreática severa $(p<0,001)$. En nuestro trabajo, la asociación entre hallazgos morfológicos parenquimatosos de pancreatitis crónica fue significativa para predecir insuficiencia pancreática exocrina. Es prometedor el rol de la ecoendoscopía para predecir el compromiso funcional de pacientes con pancreatitis crónica.

Palabras claves. Pancreatitis crónica, insuficiencia pancreática exocrina, elastasa pancreática.

\section{Rosemont Criteria in Endoscopic Ultrasound: Are They Predictors of Exocrine Pancreatic Insufficiency?}

\section{Summary}

Endoscopic ultrasonography is the method of choice for detection of morphologic changes related to chronic pancre- 
atitis. There are criteria, in the literature, that evaluate morphologic changes by endoscopic ultrasonography suggestive of chronic pancreatitis. The most widely used are the Rosemont criteria. Currently, the most effective diagnostic methods for exocrine pancreatic insufficiency are fat quantification (disused) and the 13C-mixed triglyceride breath test, not available in our country. However, the probability of diagnosis of pancreatic insufficiency can be estimated over the base of symptoms, malnutrition parameters, faecal elastase and signs of chronic pancreatitis in images. The aim of this study was to determine the correlation, probability and severity of exocrine pancreatic insufficiency, according to Rosemont criteria, in patients with chronic pancreatitis. A retrospective descriptive study was performed. Data were collected from patients between April of 2017 and April of 2018. The data gathered was based on pancreatic morphologic characteristics according to Rosemont criteria and related to the diagnosis of exocrine pancreatic insufficiency diagnosis based on the levels of stool elastase. Twenty one patients with chronic pancreatitis were included. The presence of hyperechogenic foci $>2 \mathrm{~mm}$ with posterior acoustic shadowing was associated with the presence of severe exocrine pancreatic insufficiency $(p<0.001)$. In our study, the association between parenchymal morphologic findings of chronic pancreatitis was significant in predicting exocrine pancreatic insufficiency. The role of endoscopic ultrasonography in predicting functional compromise in patients with chronic pancreatitis is promising.

Keywords. Chronic pancreatitis, exocrine pancreatic insufficiency, pancreatic elastase.

\section{Abreviaturas}

PC: Pancreatitis crónica.

USE: Ultrasonografía endoscópica.

IPE: Insuficiencia pancreática exocrina.

\section{Introducción}

La pancreatitis crónica (PC) es un síndrome caracterizado por inflamación pancreática progresiva crónica, fibrosis y cicatrización que da, como resultado, un daño persistente asociado a pérdida de células exocrinas (acinares), endocrinas (células de los islotes) y células ductales. ${ }^{1}$

El diagnóstico de PC, en estadios avanzados, suele realizarse mediante la utilización de parámetros clínicos, por la presencia de factores de riesgo y estudios complementarios por imágenes. Por otro lado, el diagnóstico de $\mathrm{PC}$ en estadios tempranos suele ser dificultoso. ${ }^{2,3}$
La ultrasonografía endoscópica (USE) es en la actualidad el método de elección para la detección de cambios morfológicos tempranos relacionados a PC, debido a su capacidad para detectar cambios menores parenquimatosos y ductales, que no pueden ser detectados por otros métodos de imágenes, y su especificidad aumenta con el aumento de los criterios diagnósticos. ${ }^{4}$ Para el diagnóstico por USE de PC, los criterios de Rosemont modificados son los más utilizados. La clasificación de Rosemont se compone de criterios mayores y menores bien definidos y cuatro grupos de diagnóstico. Los grupos de diagnóstico incluyen: consistentes, sugestivos, indeterminados y normal. La PC consistente incluye 2 criterios mayores A; o 1 mayor A + 1 mayor $\mathrm{B}$; o 1 mayor $\mathrm{A}+$ 3 criterios menores. La PC sugestiva incluye 1 criterio mayor $\mathrm{A}+<$ de 3 criterios menores; o 1 criterio mayor $\mathrm{B}+3$ criterios menores; o 5 criterios menores. La PC indeterminada comprende 1 criterio mayor $\mathrm{B}+<$ de

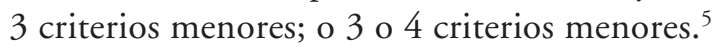

Los criterios mayores A incluyen focos hiperecoicos (con sombra acústica posterior), y cálculos del conducto mayor (estructura ecogénica). El criterio mayor B es la lobularidad ( $\geq 3$ lóbulos contiguos: "panalización”). Los criterios ductales menores incluyen: quiste, conducto dilatado, contorno irregular del conducto, rama lateral dilatada y pared del conducto hiperecoico. Los criterios parenquimatosos menores incluyen estrías hiperecoicas, focos hiperecoicos y lobularidad ${ }^{5}$ (Tabla 1).

Como consecuencia de la inflamación crónica y la fibrosis parenquimatosa del páncreas que ocurre en pacientes con PC, la insuficiencia pancreática exocrina (IPE) es la complicación más frecuente. La PC es una de las causas más frecuentes de IPE, la cual se define como el estado de maldigestión de nutrientes causado por la insuficiente secreción de enzimas pancreáticas, su inadecuada activación, su degradación precoz y/o la ineficiente interacción enzima-alimento, lo cual condiciona una malnutrición calórico-proteica y déficits vitamínicos. ${ }^{6}$

La IPE es clínicamente significativa cuando hay una reducción de la secreción de al menos el $90 \%$ de las enzimas pancreáticas, y se reporta entre el 60 y $90 \%$ de las PC dentro de los diez a doce años de evolución. ${ }^{7}$

Actualmente, la función pancreática puede ser evaluada por el coeficiente de absorción grasa (que, en la mayoría de los casos, no se realiza por falta de aceptación del método por el paciente) y el test de aliento con ${ }^{13} \mathrm{C}$ - triglicéridos, ${ }^{7}$ este último no disponible en la Argentina.

El estudio de elastasa en materia fecal es el test más sencillo y disponible, y es un indicador de función pancreática. ${ }^{8}$ 
Tabla 1. Criterios ecoendoscópicos de Rosemont para el diagnóstico de pancreatitis crónica

Criterios mayores y menores

\begin{tabular}{|c|c|c|}
\hline Parenquimatosos & $\begin{array}{l}\text { - Foco hiperecoicos } \\
\text { - Estrías hiperecoicas } \\
\text { - Lobularidad } \\
\text { - Quistes }\end{array}$ & $\begin{array}{l}\text { - Mayor A: focos hiperecoicos (> } 2 \text { mm en longitud con sombra acústica posterior). } \\
\text { - Menor: foco hiperecoico (> } 2 \text { mm de longitud sin sombra acústica posterior). } \\
\text { - Menor: estrías hiperecoicas ( } 3 \text { mm en al menos } 2 \text { direcciones diferentes con respectc } \\
\text { al plano de la imagen). } \\
\text { - Mayor B: tres lóbulos contiguos. } \\
\text { - Menor: > } 5 \text { mm, sin lóbulos contiguos. } \\
\text { - Menor: pseudoquiste/quiste (anecoico, redondo, elíptico con o sin tabiques). }\end{array}$ \\
\hline Ductales & $\begin{array}{l}\text { - Dilatación del conducto principal } \\
\text { - Irregularidad del conducto } \\
\text { - Márgenes hiperecogénicos } \\
\text { - Cálculo en el conducto principal } \\
\text { - Conductos secundarios dilatados }\end{array}$ & $\begin{array}{l}\text { - Menor: conducto dilatado ( } \geq 3,5 \mathrm{~mm} \text { en el cuerpo } 0>1,5 \mathrm{~mm} \text { en la cola), } \\
\text { - Menor: contorno irregular o irregular y curso ectásico. } \\
\text { - Menor: pared del conducto hiperecoico (estructura ecogénica, distinta > 50\% de todo } \\
\text { el conducto pancreático principal en el cuerpo y la cola). } \\
\text { - Mayor A: estructura ecogénica dentro del conducto pancreático principal con } \\
\text { sombreado acústico. } \\
\text { - Menor: > } 3 \text { estructuras anecoicas tubulares, cada una de } \geq 1 \mathrm{~mm} \text { de ancho, } \\
\text { que brotan del conducto pancreático principal. }\end{array}$ \\
\hline
\end{tabular}

Cuanto menor sea el valor de elastasa fecal, mayor será la probabilidad de IPE. Cuando el valor es menor de $100 \mathrm{ug} / \mathrm{g}$, el diagnóstico de IPE es altamente probable y cuando el valor es $100 \mathrm{ug} / \mathrm{g}-200 \mathrm{ug} / \mathrm{g}$, se recomienda considerar otras herramientas adicionales. La sensibilidad de elastasa, en materia fecal, para IPE leve, moderada y severa en pacientes con PC es del 63\%, 100\% y 100\%, respectivamente, y tiene una especificidad del $93 \%$ en pacientes con IPE. ${ }^{6}$

Nuestra hipótesis es que la USE podría proporcionar información morfológica confiable que se asocie con la probabilidad y severidad de IPE en la PC. Si se confirma dicha hipótesis, la USE podría utilizarse no solo para el diagnóstico de PC a través de los criterios de Rosemont, sino también para establecer la probabilidad de IPE y la severidad de afectación funcional (correlación morfológica-funcional). Si bien la colangiopancreatografía por resonancia magnética asociada a secretina evalúa la morfología y los cambios ductales, aún no está disponible en nuestro medio.

El objetivo de nuestro estudio es determinar la correlación, probabilidad y severidad de IPE, de acuerdo con los criterios de Rosemont, en pacientes con diagnóstico de PC.

\section{Métodos}

Diseño

Estudio retrospectivo, de corte transversal.

\section{Lugar}

Se recolectaron datos de pacientes derivados, entre abril de 2017 y abril de 2018, a la unidad de Páncreas del
Hospital Interzonal General de Agudos General José de San Martín, de La Plata.

\section{Población estudiada}

Se evaluaron las características por USE lineal multifrecuencia de 5 a $10 \mathrm{Mhz}$ bajo sedoanalgesia con equipo Pentax Avius ${ }^{\circledR}$ Hitachi, realizadas por un operador entrenado (realiza más de seiscientos procedimientos anuales), en pacientes mayores de 18 años con sospecha clínica por la presencia de dolor epigástrico y/o distensión abdominal, diarrea malaabsortiva, pérdida de peso en pacientes con factores de riesgo (antecedentes de pancreatitis, tabaquistas, enolistas) e imagenológica de PC (hallazgos morfológicos parenquimatosos y/o ductales por tomografía de abdomen con contraste o resonancia magnética de abdomen con cortes para páncreas con gadolinio). En la USE, los cambios crónicos fueron evaluados de acuerdo con la presencia de los criterios de Rosemont y se categorizó a los pacientes con PC consistente, sugestiva, e indeterminada. Posteriormente, estos criterios se relacionaron con el diagnóstico y la severidad de IPE. El diagnóstico de IPE se determinó de acuerdo con los valores de elastasa en materia fecal, a través de Elisa Kit (ScheBo ${ }^{\circledR}$ Pancreatic Elastase), que utiliza anticuerpos monoclonales. Las muestras de heces se recolectaron en el hogar o en el hospital. Se recibió la muestra el mismo día y se almacenó inmediatamente a $-20^{\circ} \mathrm{C}$.

Se consideró IPE severa cuando los valores de elastasa fueron menores a $100 \mu \mathrm{g} / \mathrm{g}$, y leve a moderada cuando fueron entre 100 a $200 \mu \mathrm{g} / \mathrm{g}$. 


\section{Metodología estadística empleada}

Los datos categóricos y continuos fueron presentados como proporción, media y desvío estándar, respectivamente.

La metodología estadística utilizada para comparar criterios de Rosemont, con probabilidad de IPE, fue el test de Fisher, y para evaluar severidad de IPE, se utilizó el test de Kruskal Wallis con corrección de Bonferroni. Se consideró significativa una $p<0,05$. Se utilizó el programa STATA (Stata Corp., College Station, Texas, EE. UU.) versión 14.0.

\section{Resultados}

Se analizaron los datos de veintiún pacientes con diagnóstico de PC, de acuerdo con los criterios de Rosemont. Según estos criterios, ocho pacientes (38\%) cumplían los criterios de pancreatitis crónica consistente, tres (14\%) pancreatitis crónica sugestiva y diez (47\%) pancreatitis indeterminada. Se incluyeron siete $(33 \%)$ pacientes de sexo femenino y catorce $(66 \%)$ de sexo masculino, con una media de edad de 51 años (rango de 45 a 64), once (52\%) de ellos con diagnóstico de IPE severa.

La presencia de focos hiperecoicos > de $2 \mathrm{~mm}$ con sombra acústica posterior (calcificaciones parenquimatosas) se asoció a la presencia de IPE severa en el $81 \%$ de los casos (nueve pacientes), en forma significativa $(p<0,001)$ (Figura 1).

Se observó una tendencia hacia la presencia de litiasis intraductal (Figura 2) y dilatación del conducto pancreático principal en los pacientes con IPE (Figura 3), pero dicha tendencia no fue estadísticamente significativa $(p<0,2)$.

Figura 1. USE lineal: litiasis intrapancreática $>2 \mathrm{~mm}$ con sombra acústica posterior

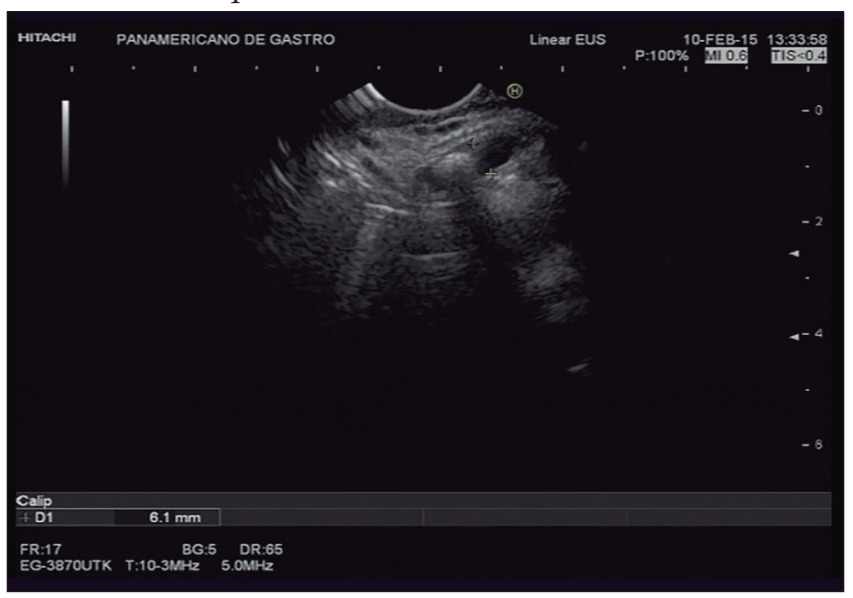

Figura 2. USE lineal: presencia de cálculos en el conducto pancreático principal

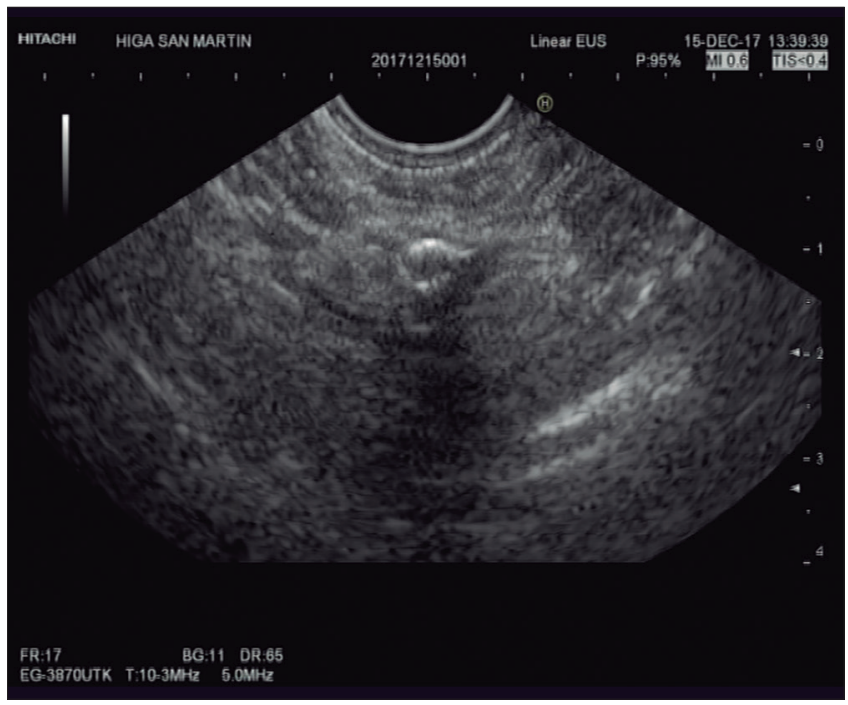

Figura 3. USE lineal: conducto pancreático principal dilatado

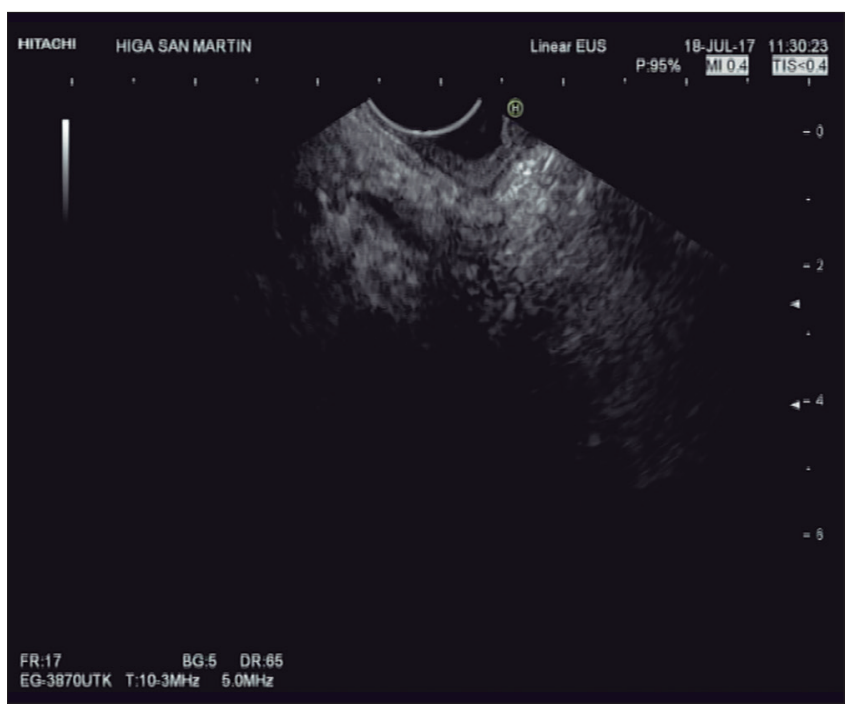

El número de criterios de Rosemont fue significativamente mayor en pacientes con PC e IPE (cuatro criterios) en comparación a pacientes con diagnóstico de PC, pero que no tenían diagnóstico de IPE (tres criterios) $(p<0,04)$.

Los pacientes con PC consistente, de acuerdo con los criterios de Rosemont, tuvieron una media de cinco criterios de Rosemont, en comparación con los pacientes con pancreatitis indeterminada, quienes tuvieron una media de criterios de tres $(p<0,001)$.

De los ocho pacientes en el grupo de pancreatitis consistente, la media de elastasa, en materia fecal, fue 
de $31 \mu \mathrm{g} / \mathrm{g}(14-59 \mu \mathrm{g} / \mathrm{g})$, en los pacientes con pancreatitis sugestiva fue de $226 \mu \mathrm{g} / \mathrm{g}(38-336 \mu \mathrm{g} / \mathrm{g})$, y en los diez pacientes con pancreatitis indeterminada la media de los valores de elastasa en materia fecal fue de $368 \mu \mathrm{g} / \mathrm{g}$
(310-510 $\mu \mathrm{g} / \mathrm{g})$. Las diferencias de estos valores fueron significativas en la comparación del grupo de pancreatitis consistente vs. pancreatitis indeterminada $(p<0,003)$ (Gráfico 1).

Gráfico 1. Valores de elastasa fecal de acuerdo a criterios de Rosemont

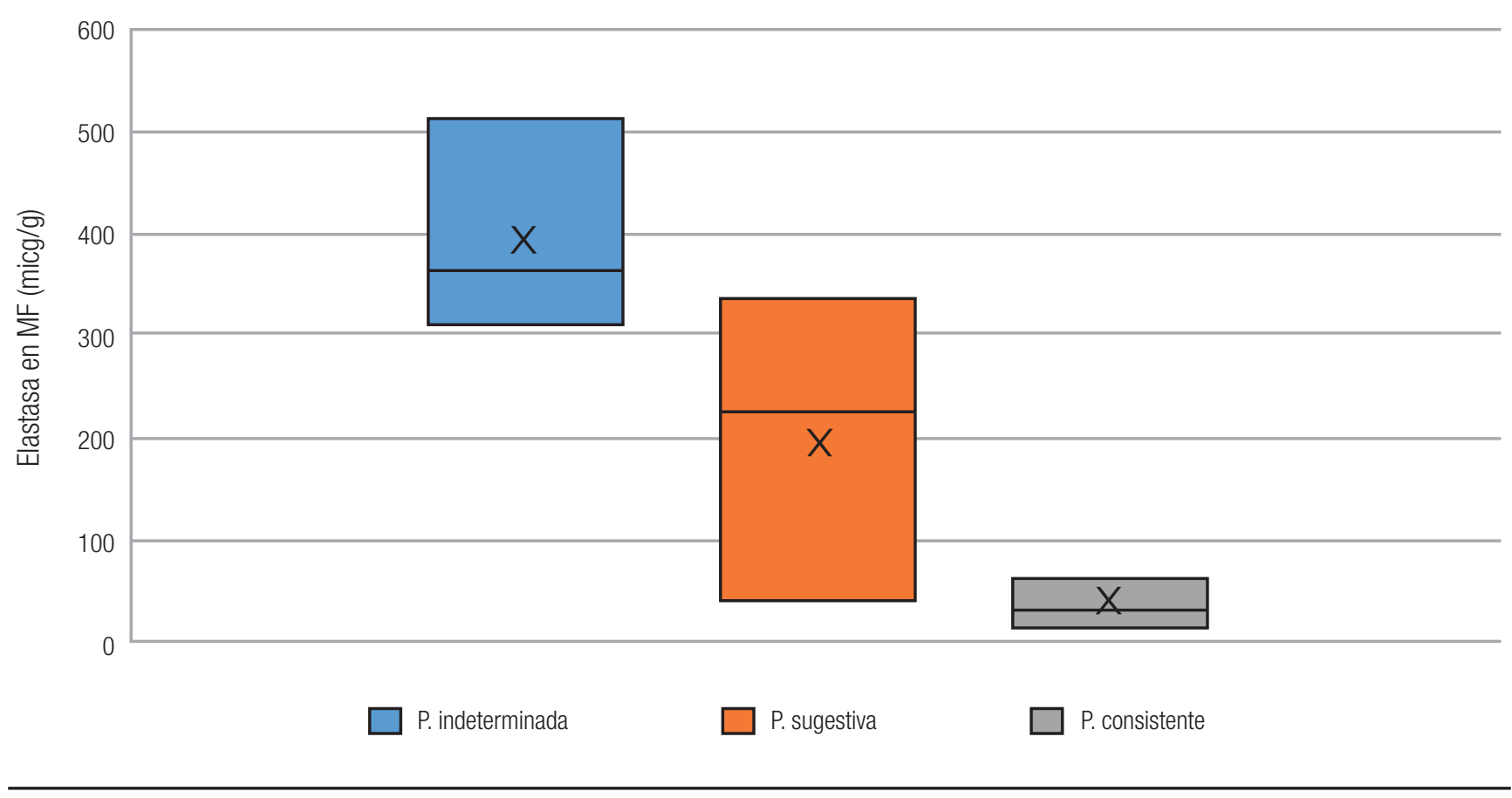

\section{Discusión}

La IPE es la alteración de la función pancreática, causada por la insuficiente secreción de enzimas pancreáticas, su inadecuada activación, su degradación precoz y/o la ineficiente interacción enzima-alimento, lo cual condiciona una malnutrición calórico-proteica y déficits vitamínicos. ${ }^{6}$ La malnutrición secundaria a IPE se asocia con complicaciones, como aumento de la probabilidad de eventos cardiovasculares, que se han relacionado con niveles plasmáticos anormalmente bajos de lipoproteína $\mathrm{C}$ de alta densidad, apolipoproteína A-I y lipoproteína A. ${ }^{9}$

Las causas de IPE pueden ser primarias o pancreáticas por déficit de secreción (PC, pancreatitis recurrente o aguda, fibrosis quística, esteatosis pancreática, diabetes, tumores pancreáticos y periampulares, y cirugías pancreáticas), o secundarias (extrapancreáticas), como inadecuado aporte calórico-proteico, enfermedad celíaca, enfermedad de Crohn, linfomas, tumores neuroendócrinos, virus de la inmunodeficiencia humana adquirida y cirugías gastroduodenales. ${ }^{6}$ Se la considera una enfermedad infradiagnosticada, siendo la etiología más frecuente la PC.

Los criterios de Rosemont son los más utilizados para el diagnóstico de PC, y permiten clasificar los hallazgos ecoendoscópicos en cuatro grupos de diagnóstico (consistentes, sugestivos, indeterminados y normal), según la presencia de criterios mayores o menores. ${ }^{5}$

En este trabajo, se analizaron los datos de veintiún pacientes con diagnóstico de PC (de diversas etiologías) de acuerdo con los criterios de Rosemont. El 47\% de los pacientes (diez pacientes) cumplían los criterios de pancreatitis indeterminada, el 38\% (ocho pacientes), cumplían los criterios de pancreatitis consistente y $14 \%$ (tres pacientes) tenían criterios de pancreatitis crónica sugestiva.

Los pacientes con PC e IPE deben ser tratados aun en ausencia de síntomas, ya que los pacientes tienden a limitar la ingesta de grasas de forma inconsciente y progresiva; por este motivo, la diarrea no siempre está presente en pacientes con una inadecuada digestión de grasas, lo que 
apoya el concepto de que los síntomas no son fiables para el diagnóstico de IPE. ${ }^{8}$

Para el diagnóstico de IPE, el coeficiente de cuantificación de la absorción de grasa sigue siendo el método gold standard, pero esta prueba tiene importantes limitaciones: requiere que los pacientes sigan una dieta estricta (100 g de grasa al día durante cinco días) y la recolección total de heces excretadas durante los últimos tres días. Por tales motivos, esta prueba está en desuso. ${ }^{8}$ En la práctica clínica, la determinación de elastasa en materia fecal y el estudio de biomarcadores séricos son los métodos de elección por su sencillez y disponibilidad. ${ }^{6}$

En este estudio, se demostró que la correlación entre hallazgos morfológicos parenquimatosos y ductales de PC fue significativa para predecir IPE. La presencia de focos hiperecoicos de $>2 \mathrm{~mm}$ con sombra acústica posterior (calcificaciones parenquimatosas) se asoció a la presencia de IPE severa en el $81 \%$ de los casos (nueve pacientes). Se observó una tendencia hacia la presencia de litiasis intraductal y dilatación del conducto pancreático principal en los pacientes con IPE, pero dicha tendencia no fue estadísticamente significativa $(p<0,2)$, aunque inferimos que fue por el limitado número de pacientes. Se evidenció que la cantidad de criterios de Rosemont en pacientes con PC e IPE fue mayor (cuatro criterios) en comparación con los pacientes que tenían diagnóstico de PC sin IPE (tres criterios), y esta diferencia fue estadísticamente significativa $(p<0,04)$.

En concordancia con publicaciones anteriores, ${ }^{5,9}$ este estudio demuestra que existe una correlación entre los hallazgos morfológicos evidenciados por USE y la probabilidad de IPE. Enrique Domínguez Muñoz y colaboradores, en el año 2012, ${ }^{9}$ analizaron la probabilidad de IPE (diagnosticada por el test de aire espirado que utiliza triglicéridos marcados con C13), de acuerdo con los criterios ecoendoscópicos de PC. Este estudio determinó que la probabilidad de IPE, en presencia de cálculos en el conducto pancreático principal, es del $80 \%$ y aumenta al $82,8 \%$ si, además, el conducto pancreático principal está dilatado.

Otro estudio de similares características, publicado por Sinan Akay y colaboradores en el año 2018, ${ }^{5}$ determinó que los valores de elastasa, en materia fecal, pueden predecir cambios crónicos en el páncreas. Del mismo modo, en nuestro estudio, se evidenció que la media de elastasa en materia fecal varía de acuerdo con las categorías de Rosemont, siendo mucho menor en el grupo de PC consistente $(31 \mu \mathrm{g} / \mathrm{g})$, en comparación con $\mathrm{PC}$ indeterminada $(368 \mu \mathrm{g} / \mathrm{g})$ siendo la diferencia significativa $(p<0,003)$.

Las ventajas de este estudio es que todas las USE fue- ron realizadas por el mismo operador y con el mismo equipo. El método que utilizamos para el dosaje de elastasa en materia fecal basado en anticuerpos monoclonales posee mayor especificidad en comparación con ensayos con anticuerpos policlonales, que sobrestiman la concentración de elastasa. Además, el ensayo monoclonal no es afectado por la terapia de reemplazo enzimático. ${ }^{5,10}$

La alta precisión y el amplio uso de la USE, para el diagnóstico de PC, hacen especialmente interesante su utilidad para la evaluación indirecta de la probabilidad de IPE sobre la base de hallazgos morfológicos, al momento de hacer el diagnóstico de la enfermedad.

Las principales limitaciones de nuestro estudio son: el tamaño muestral pequeño, la realización del mismo en un único centro y la utilización de elastasa fecal para el diagnóstico de IPE, por la falta de disponibilidad en el país del test de aliento con triglicéridos marcados con C13, el cual es considerado el gold standard para el diagnóstico de IPE.

\section{Conclusión}

En nuestro estudio se observó:

- La presencia de calcificaciones parenquimatosas se asoció a la presencia de IPE severa.

- La cantidad de criterios de Rosemont es mayor en pacientes con diagnóstico de PC e IPE, en comparación con los pacientes con PC sin diagnóstico de IPE.

- Los valores de elastasa fecal son menores en los pacientes con PC consistente, en comparación con los pacientes con PC indeterminada.

La USE es, en la actualidad, el método por imágenes más preciso para el diagnóstico de PC temprana. Si bien faltan estudios prospectivos y de mayor número de pacientes para poder generalizar estos resultados, es prometedor el rol de la USE para predecir el compromiso funcional de pacientes con PC.

Sostén financiero. No se recibieron becas ni otros aportes económicos para la realización del estudio.

\footnotetext{
Aviso de derechos de autor

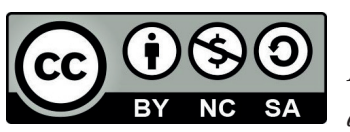

(C) 2021 Acta Gastroenterológica Latinoamericana. Este es un artículo de acceso abierto publicado bajo los términos de la Licencia Creative Commons Attribution (CC BY-NC-SA 4.0), la cual permite el uso, la distribución $y$ la reproducción de forma no comercial, siempre que se cite al autor y la fuente original.
} 
Cite este artículo como: Arramón $M$, Redondo A, Curay P $y$ col. Los criterios de Rosemont en ultrasonografía endoscópica: ¿son predictores de insuficiencia pancreática exocrina? Acta Gastroenterol Latinoam. 2021;51(2):189-95. https:// doi.org/10.52787/qwpo5827

\section{Referencias}

1. Pham A, Forsmark C. Chronic pancreatitis: review and update of etiology, risk factors, and management. F1000Res [Internet]. 2018;7.

2. Whitcomb DC, Shimosegawa T, Chari ST, Forsmark CE, Frulloni L, Garg P, et al. International consensus statements on early chronic Pancreatitis. Recommendations from the working group for the international consensus guidelines for chronic pancreatitis in collaboration with The International Association of Pancreatology, American Pancreatic Association, Japan Pancreas Society, PancreasFest Working Group and European Pancreatic Club. Pancreatology. 2018;18(5):516-27.

3. Löhr JM, Domínguez-Muñoz E, Rosendahl J, Besselink M, Mayerle J, Lerch MM, et al. United European Gastroenterology evidence-based guidelines for the diagnosis and therapy of chronic pancreatitis (HaPanEU). United European Gastroenterol J. 2017;5(2):153-99.

4. Jimeno-Ayllón C, Pérez-García JI, Gómez-Ruiz CJ, García-Cano-Lizcano J, Morillas-Ariño J, Martínez-Fernández R, Serrano-Sánchez L, Pérez-Sola Á. Standard criteria versus Rosemont classification for EUS-diagnosis of chronic pancreatitis. Rev Esp Enferm Dig. 2011;103(12):626-31
5. Akay S, Şirin B, Ünsal B. Fecal Elastase Levels Predict Honeycombing in Pancreas Detected with Endoscopic Ultrasound. Can J Gastroenterol Hepatol. 2018;2018:4625247.

6. Basso S, Canicoba M, Capitanich P, Carballido M, Crisci J, Della Gisutina F, Granero L, Gutiérrez SC, Hwang HJ, Jerez I, Kohan G, Linares ME, Marchi L, Martini G, Mazza O, Moro M, Palavecino M, Pasqua A, Uranga G. Consenso argentino de diagnóstico y tratamiento de la insuficiencia pancreática exocrina. Acta Gastroenterol Latinoam. 2018;48(3):213-25.

7. Capurso G, Traini M, Piciucchi M, Signoretti M, Arcidiacono PG. Exocrine pancreatic insuffciency: prevalence, diagnosis, and management. Clin Exp Gastroenterol. 2019;12:129-39.

8. Domínguez-Muñoz JE. Diagnosis and treatment of pancreatic exocrine insufficiency. Curr Opin Gastroenterol. 2018;34(5):349-54.

9. Domínguez-Muñoz JE, Álvarez-Castro A, Lariño-Noia J, Nieto L, Iglesias-García J. Endoscopic ultrasonography of the pancreas as an indirect method to predict pancreatic exocrine insufficiency in patients with chronic pancreatitis. Pancreas. 2012;41(5):724-8.

10. Hardt PD, Hauenschild A, Nalop J, Marzeion AM, Porsch-Ozcürümez M, Luley C, Sziegoleit A, Kloer HU. The commercially available ELISA for pancreatic elastase 1 based on polyclonal antibodies does measure an as yet unknown antigen different from purified elastase 1. Binding studies and clinical use in patients with exocrine pancreatic insufficiency. Z Gastroenterol. 2003;41(9):903-6. 